\title{
Immunohistochemical Detection of Tumor Necrosis Factor Alpha in Ileocolonic Biopsies of Patients with Inflammatory Bowel Disease Ehab Ahmed Abdelatti ${ }^{1}$, Alsaid Ibrahim Alshayeb ${ }^{1}$, Asmaa Gaber Abdou' ${ }^{2}$ Ezzat Mohamed Abdalla*1, Ezz El-Arab A. ${ }^{1}$ \\ Departments of ${ }^{1}$ Internal Medicine (Gastroenterology and Hepatology Unit) and ${ }^{2}$ Pathology, Faculty of Medicine, Menoufia University, Egypt \\ *Corresponding author: Ezzat Mohamed Abdalla, Mobile: (+20) 01001131318, Email: eabdalla89@yahoo.com
}

\begin{abstract}
Background: Tumor necrosis factor alpha (TNF- $\alpha$ ) is a major proinflammatory cytokine playing paramount role in the pathophysiology of inflammatory bowel disease (IBD). The identification of predictive biomarkers to establish response to treatment is intuitive and still unresolved need. A biomarker of TNF- $\alpha$ sensitivity would avoid unnecessary exposure of the patient to the drug and to its side effects.

Objective: To investigate the relationship between immunohistochemical expression of TNF- $\alpha$ in the biopsies of ileocolonic segments in patients with activity of both ulcerative colitis (UC) and Crohn's disease (CD) and the clinicopathologic parameters and the response to treatment.

Patients and methods: The study was conducted on 41 naïve patients diagnosed as IBD (UC and CD) and 15 normal subjects as a control group. All patients and control underwent full history taking (age, gender and clinical data as weight loss, diarrhea, bleeding, and duration of the disease), complete physical examination, laboratory investigation (Complete blood count (CBC), ESR, C-reactive protein (CRP), fecal calprotectin (FC)), colonoscopy disease localization, upper endoscope with upper GIT symptoms. Biopsies from the terminal ileum and different colon segments were taken for histopathological evaluation and immunohistochemistry was done according to published protocols to all cases and control. Results: Percentage and H-score for TNF- $\alpha$ significantly correlated with clinical severity and need to give additional treatment besides conventional therapy as anti-TNF therapy. Increased TNF- $\alpha$ expression is significantly associated with better response to anti-TNF treatment.

Conclusion: Increased expression of TNF- $\alpha$ in the tissues is associated with the need to give additional treatment besides conventional therapy as anti-TNF therapy and also increased TNF expression is associated with better response to anti-TNF treatment.
\end{abstract}

Keywords: Anti-TNF treatment, Inflammatory bowel disease, Tumor necrosis factor alpha.

\section{INTRODUCTION}

Tumor necrosis factor alpha (TNF- $\alpha)$ is a major proinflammatory cytokine playing important role in the pathophysiology of IBD (1), and blocking its inflammatory pathway leads to effective control of the disease ${ }^{(2)}$. The identification of predictive biomarkers to establish response to treatment is intuitive and still unresolved need. A biomarker of TNF- $\alpha$ sensitivity would avoid unnecessary exposure of the patient to the drug and to its side effects ${ }^{(3)}$.

Unfortunately, apart from the demonstration that patients with high blood levels of CRP respond better to anti-TNF $\alpha$ agents, no other biomarkers are available in the daily routine ${ }^{(4)}$. A study performed on UC patients employing immunohistochemical techniques showed that TNF $\alpha$ expression correlates to the degree of colonic inflammation, and that tissue levels of TNF $\alpha$ may predict response to infliximab treatment ${ }^{(5)}$. Moreover, in patients with $\mathrm{CD}$, high expression of TNF- $\alpha$ in the inflamed ileocolonic tissues of patients may predict the response to biological agents (9).The aim of the present study was to investigate the relationship between immunohistochemical expression of TNF- $\alpha$ in the biopsies of ileocolonic segments in patients with activity of both ulcerative colitis (UC) and
Crohn's disease (CD) and the clinicopathologic parameters and the response to treatment.

\section{PATIENTS AND METHODS}

The study was conducted on 41 naïve patients diagnosed as inflammatory bowel disease (IBD) (UC and CD) from the GI Endoscopy Unit of Internal Medicine Department, Menoufia University Hospital. 15 normal subjects were recruited as a control group. All patients and control underwent full history taking (age, gender and clinical data as weight loss, diarrhea, bleeding, and duration of the disease), complete physical examination, laboratory investigations including (CBC, ESR, CRP, FC), colonoscopy disease localization and assessment, upper endoscope in cases with upper GIT symptoms, and assessment of clinical severity.

Biopsies from the terminal ileum and different colon segments were taken for histopathological evaluation. The specimens were processed in Pathology Department for embedding in paraffin. From each paraffin embedded block, $5 \mu \mathrm{m}$-thick sections were cut and stained by haematoxylin and eosin ( $\mathrm{H}$ and $\mathrm{E})$ to confirm the diagnosis and to assess the histopathologic severity by original Geboes score in confirmed UC cases ${ }^{(7)}$. Immunohistochemistry was done according to 
published protocols to all cases and control. Expression, intensity, percentage and $\mathrm{H}$ score were reported ${ }^{\left({ }^{(8)}\right.}$. All patients received treatment according to the guidelines and response to the received treatment (clinical and endoscopic) was assessed 3-6 months after receiving the treatment whether the patient received traditional or conventional treatment (anti-inflammatory, steroids, immunosuppressives) or anti TNF- $\alpha$ therapy.

\section{Ethical approval:}

This study was conducted after approval of the Ethical Committee Board, Menoufia University and informed written consent from each patient was obtained.

\section{Statistical analysis}

Data were collected, tabulated and statistically analyzed using an IBM compatible personal computer with Statistical Package for the Social Sciences (SPSS) version 23 (SPSS Inc. Released 2015. IBM SPSS statistics for windows, version 23.0, Armnok, NY: IBM Corp.). Descriptive statistics e.g., qualitative data were expressed in: Number $(\mathrm{N})$, percentage (\%), while quantitative data were expressed as mean, standard deviation (SD), median, and range (minimummaximum). Independent t-test, Mann Whitney's test, ANOVA test, and Kruskal Wallis test were used for comparison of quantitative variables. Tamhane test was used as the post-hoc of Kruskal Wallis test, while LSD was the post-hoc test of ANOVA test. Chi-square test $\left(\chi^{2}\right)$ was used to study association between qualitative variables. Whenever any of the expected cells were less than five, Fischer's exact test was used. Pearson correlation was used to show correlation between two continuous variables. Significant test results were quoted as two-tailed probabilities. Significance of the obtained results was judged at the 5\% level $(\mathrm{P}<0.05)$.

\section{RESULTS}

As regards the clinicopathological data, there was no significant difference among UC, CD and control regarding the age, but there was a significant difference regarding gender, $\mathrm{CRP}, \mathrm{Hb}, \mathrm{ESR}$ and fecal calprotectin (Table 1).

Table (1): Differences among UC, CD and control regarding clinicopathological data

\begin{tabular}{|c|c|c|c|c|c|c|c|c|}
\hline $\begin{array}{c}\text { Clinicopathological } \\
\text { data }\end{array}$ & \multicolumn{2}{|c|}{$\begin{array}{c}\mathrm{UC} \\
(\mathrm{N}=26)\end{array}$} & \multicolumn{2}{|c|}{$\begin{array}{c}\mathrm{CD} \\
(\mathrm{N}=15)\end{array}$} & \multicolumn{2}{|c|}{$\begin{array}{c}\text { Control } \\
(\mathrm{N}=15)\end{array}$} & $\begin{array}{l}\text { Test of } \\
\text { sig. }\end{array}$ & $\begin{array}{c}\text { P value } \\
\text { (Post-Hoc) }\end{array}$ \\
\hline $\begin{array}{l}\text { Age (years) } \\
\text { Mean } \pm \text { SD } \\
\text { Median } \\
\text { Range }\end{array}$ & \multicolumn{2}{|c|}{$\begin{array}{c}31.6 \pm 11.7 \\
28.5 \\
18-57\end{array}$} & \multicolumn{2}{|c|}{$\begin{array}{c}30 \pm 19.01 \\
20 \\
16-70\end{array}$} & \multicolumn{2}{|c|}{$\begin{array}{c}34 \pm 13.1 \\
30 \\
23-65\end{array}$} & $\begin{array}{c}\mathbf{K}-\mathbf{W}= \\
10.7\end{array}$ & 0.08 \\
\hline $\begin{array}{l}\text { Gender: } \\
\text { Male } \\
\text { Female }\end{array}$ & $\begin{array}{c}8 \\
18\end{array}$ & $\begin{array}{l}30.8 \% \\
69.2 \%\end{array}$ & $\begin{array}{c}12 \\
3\end{array}$ & $\begin{array}{l}80 \% \\
20 \%\end{array}$ & $\begin{array}{l}7 \\
8\end{array}$ & $\begin{array}{l}46.7 \% \\
53.3 \%\end{array}$ & $\begin{array}{l}\mathbf{X}^{2}= \\
9.2\end{array}$ & $\begin{array}{c}\text { O.01* } \\
\text { P1:0.002 } \\
\text { P2:0.309 } \\
\text { P3:0.058 } \\
\end{array}$ \\
\hline \multicolumn{9}{|l|}{ Investigations } \\
\hline $\begin{array}{l}\text { CRP(mg/dl) } \\
\text { Positive } \\
\text { Negative }\end{array}$ & $\begin{array}{c}21 \\
5\end{array}$ & $\begin{array}{l}80.8 \% \\
19.2 \%\end{array}$ & $\begin{array}{c}10 \\
5\end{array}$ & $\begin{array}{l}66.7 \% \\
33.3 \%\end{array}$ & $\begin{array}{c}4 \\
11\end{array}$ & $\begin{array}{l}26.7 \% \\
73.3 \%\end{array}$ & $\begin{array}{c}\mathbf{X}^{2}= \\
12.03\end{array}$ & $\begin{array}{c}\text { 0.002* } \\
\text { P1:0.311 } \\
\text { P2:0.001 } \\
\text { P3:0.028 }\end{array}$ \\
\hline $\begin{array}{l}\text { Mean } \pm \text { SD } \\
\text { Median }\end{array}$ & \multicolumn{2}{|c|}{$\begin{array}{c}19.3 \pm 2.9 \\
17.5\end{array}$} & \multicolumn{2}{|c|}{$\begin{array}{c}21.5 \pm 4.01 \\
30\end{array}$} & \multicolumn{2}{|c|}{$\begin{array}{c}4.9 \pm 1.2 \\
5\end{array}$} & $\begin{array}{c}\mathbf{K}-\mathbf{W}= \\
13.9\end{array}$ & $\begin{array}{c}\text { 0.001* } \\
\text { P1:0.955 } \\
\text { P2: }<0.001 \\
\text { P3:0.004 }\end{array}$ \\
\hline $\begin{array}{l}\mathbf{H b}(\mathbf{g} / \mathbf{d L}) \\
\text { Mean } \pm \text { SD } \\
\text { Median }\end{array}$ & \multicolumn{2}{|c|}{$\begin{array}{c}10.3 \pm 1.9 \\
10.7\end{array}$} & \multicolumn{2}{|c|}{$\begin{array}{l}9.6 \pm 2.2 \\
9.5\end{array}$} & \multicolumn{2}{|c|}{$\begin{array}{c}12.1 \pm 0.6 \\
12\end{array}$} & $\begin{array}{c}\text { ANOVA } \\
8.4\end{array}$ & $\begin{array}{c}\text { 0.001* } \\
\text { P1:0.207 } \\
\text { P2:0.003 } \\
\text { P3: }<\text { 0.001 } \\
\end{array}$ \\
\hline \begin{tabular}{|l|}
$\mathbf{E S R}(\mathbf{m m} / \mathbf{h})$ \\
Mean \pm SD \\
Median
\end{tabular} & \multicolumn{2}{|c|}{$\begin{array}{c}39.1 \pm 9.6 \\
30\end{array}$} & \multicolumn{2}{|c|}{$\begin{array}{c}37.3 \pm 3.2 \\
30\end{array}$} & \multicolumn{2}{|c|}{$\begin{array}{c}5.9 \pm 1.3 \\
6\end{array}$} & $\begin{array}{c}\mathbf{K}-\mathbf{W}= \\
30.92\end{array}$ & $\begin{array}{c}<<.001 * * \\
\text { P1:0.996 } \\
\text { P2: }<0.001 \\
\text { P3: }<0.001\end{array}$ \\
\hline $\begin{array}{l}\text { Fecal calprotectin } \\
(\text { mcg/g) } \\
\text { Mean } \pm \text { SD } \\
\text { Median }\end{array}$ & \multicolumn{2}{|c|}{$\begin{array}{c}440.8 \pm 36.96 \\
375\end{array}$} & \multicolumn{2}{|c|}{$\begin{array}{c}340 \pm 26.1 \\
300\end{array}$} & \multicolumn{2}{|c|}{$\begin{array}{c}61.3 \pm 8.8 \\
60\end{array}$} & $\begin{array}{l}\mathbf{K}-\mathbf{W}= \\
23.47\end{array}$ & $\begin{array}{c}<0.001 * * \\
\text { P1:0.595 } \\
\text { P2: }<0.001 \\
\text { P3:0.001 } \\
\end{array}$ \\
\hline
\end{tabular}

$\mathrm{K}-\mathrm{W}$ : Kruskal-Wallis test, * significant, **highly significant,

P1: between UC and CD, P2: between UC and control, P3: between CD and control. Moreover, there was a significant difference among UC, CD and control regarding TNF- $\alpha$ expression, percentage of positive inflammatory cells and $\mathrm{H}$ score, but there was no statistically significant difference regarding TNF- $\alpha$ staining intensity (Table 2 ). 
Table (2): Differences among ulcerative colitis (UC), Crohn's disease (CD) and control groups regarding TNF$\alpha$ immunostaining results

\begin{tabular}{|c|c|c|c|c|c|c|c|c|}
\hline \multirow[b]{2}{*}{ TNF- $\alpha$ data } & \multicolumn{2}{|c|}{$\mathrm{UC}(\mathrm{N}=26)$} & \multicolumn{2}{|c|}{ CD $(\mathrm{N}=15)$} & \multicolumn{2}{|c|}{ Control $(\mathrm{N}=15)$} & \multirow{2}{*}{$\begin{array}{l}\text { Test } \\
\text { of sig. }\end{array}$} & \multirow[b]{2}{*}{$P$ value } \\
\hline & $\mathbf{N}$ & $\%$ & $\mathbf{N}$ & $\%$ & $\mathbf{N}$ & $\%$ & & \\
\hline $\begin{array}{l}\text { TNF- } \alpha \text { expression: } \\
\text { Positive } \\
\text { Negative }\end{array}$ & $\begin{array}{c}26 \\
0\end{array}$ & $\begin{array}{c}100 \% \\
0 \%\end{array}$ & $\begin{array}{c}15 \\
0\end{array}$ & $\begin{array}{c}100 \% \\
0 \%\end{array}$ & $\begin{array}{c}4 \\
11\end{array}$ & $\begin{array}{l}26.7 \% \\
73.3 \%\end{array}$ & $\begin{array}{l}\mathbf{X}^{2}= \\
37.4\end{array}$ & $\begin{aligned} r<0.001 * * \\
\text { P1: } 1 \\
\text { P2: }<00.001 \\
\text { P3: }<0.001 \\
\end{aligned}$ \\
\hline $\begin{array}{l}\text { Staining intensity: } \\
\text { Mild } \\
\text { Moderate } \\
\text { Strong }\end{array}$ & $\begin{array}{c}9 \\
10 \\
7 \\
\end{array}$ & $\begin{array}{l}34.6 \% \\
38.5 \% \\
26.9 \% \\
\end{array}$ & $\begin{array}{l}5 \\
4 \\
6 \\
\end{array}$ & $\begin{array}{c}33.3 \% \\
26.7 \% \\
40 \% \\
\end{array}$ & $\begin{array}{l}3 \\
1 \\
0 \\
\end{array}$ & $\begin{array}{l}75 \% \\
25 \% \\
0 \% \\
\end{array}$ & $\begin{array}{l}\mathbf{X}^{2}= \\
3.93\end{array}$ & 0.415 \\
\hline $\begin{array}{l}\text { Percent of positive } \\
\text { inflammatory cells }(\%) \text { : } \\
\text { Number } \\
\text { Mean } \pm \text { SD } \\
\text { Median } \\
\end{array}$ & & $\begin{array}{l}26 \\
2 \pm 20.4 \\
60 \\
\end{array}$ & & $\begin{array}{l}15 \\
7 \pm 10.5 \\
60 \\
\end{array}$ & & $\begin{array}{c}4 \\
8.3 \pm 7.9 \\
5\end{array}$ & $\begin{array}{c}\mathrm{K}-\mathrm{W}= \\
13.8\end{array}$ & $\begin{array}{l}\text { P.001* } \\
\text { P1:0.103 } \\
\text { P2: }<0.001 \\
\text { P3: }<0.001 \\
\end{array}$ \\
\hline $\begin{array}{l}\text { H-score: } \\
\text { Number } \\
\text { Mean } \pm \text { SD } \\
\text { Median } \\
\end{array}$ & & $\begin{array}{l}26 \\
7 \pm 80.5 \\
90 \\
\end{array}$ & & $\begin{array}{l}15 \\
.3 \pm 69.1 \\
140 \\
\end{array}$ & & $\begin{array}{c}4 \\
3.3 \pm 17.9 \\
5 \\
\end{array}$ & $\begin{array}{c}\mathrm{K}-\mathrm{W}= \\
32.6\end{array}$ & $\begin{array}{l}<0.001 * * \\
\text { P1:0.8 } \\
\text { P2: }<0.001 \\
\text { P3: }<0.001 \\
\end{array}$ \\
\hline
\end{tabular}

K-W: Kruskal-Wallis test, * significant, **highly significant

P1: between UC and CD, P2: between UC and control, P3: between CD and control.

The percentage of TNF- $\alpha$ expressing inflammatory cells positively correlated with levels of CRP, ESR, and fecal calprotectin, while it was negatively correlated with $\mathrm{Hb}$ level (Table 3 ).

Table (3): Correlation between percentage of positive inflammatory cells expressing TNF- $\alpha$ in IBD cases and CRP, Hb, ESR, and fecal calprotectin

\begin{tabular}{|l|c|c|}
\hline \multicolumn{1}{|c|}{$\begin{array}{c}\text { Clinicopathological } \\
\text { data }\end{array}$} & \multicolumn{2}{c|}{ Percentage of TNF- $\boldsymbol{\alpha}$ in inflammatory bowel disease cases } \\
\cline { 2 - 3 } & Test of significance & P value \\
\hline CRP $(\mathbf{m g} / \mathbf{d L})$ & $\mathrm{r}=0.47$ & $\mathbf{0 . 0 0 2}$ \\
\hline Hb (gm/dL) & $\mathrm{r}=-0.46$ & $\mathbf{0 . 0 0 2} *$ \\
\hline ESR $(\mathbf{m m} / \mathbf{h})$ & $\mathrm{r}=0.48$ & $\mathbf{0 . 0 0 2}$ \\
\hline Fecal calprotectin $(\mathbf{m c g} / \mathrm{g})$ & $\mathrm{r}=0.58$ & $<\mathbf{0 . 0 0 1 * *}$ \\
\hline
\end{tabular}

r: Pearson correlation coefficient* significant, **highly significant

There was significant progressive increase in the percentage of positive inflammatory cells expressing TNF- $\alpha$ with the clinical severity and given treatment (Table 4).

Table (4): Comparison between percentage of positive inflammatory cells expressing TNF- $\alpha$ in IBD cases as regard clinical severity and treatment received

\begin{tabular}{|c|c|c|c|c|}
\hline \multirow{2}{*}{ Clinicopathological data } & \multicolumn{2}{|c|}{$\begin{array}{c}\text { Percentage of TNF- } \alpha \text { in } \\
\text { inflammatory bowel disease cases }\end{array}$} & \multirow{2}{*}{$\begin{array}{c}\text { Test of } \\
\text { significance }\end{array}$} & \multirow[t]{2}{*}{ P-value } \\
\hline & $\mathbf{N}$ & Mean \pm SD & & \\
\hline $\begin{array}{l}\text { Clinical severity: } \\
\text { Mild } \\
\text { Moderate } \\
\text { Severe } \\
\text { Fulminant }\end{array}$ & $\begin{array}{c}14 \\
16 \\
9 \\
2\end{array}$ & $\begin{array}{c}46.4 \pm 15.9 \\
63.7 \pm 15.4 \\
67.8 \pm 10.9 \\
90\end{array}$ & $\mathrm{~K}-\mathrm{W}=15.012$ & $\begin{array}{r}\text { O.002** } \\
\text { P1: } 0.033 \\
\text { P2: } 0.006 \\
\text { P3: }<0.001 \\
\text { P4:0.974 } \\
\text { P5: }<0.001 \\
\text { P6: } 0.002\end{array}$ \\
\hline $\begin{array}{l}\text { Treatment: } \\
\text { Traditional } \\
\text { Anti-TNF therapy }\end{array}$ & $\begin{array}{l}26 \\
15 \\
\end{array}$ & $\begin{array}{c}53.1 \pm 17.4 \\
72 \pm 12.1 \\
\end{array}$ & $\mathrm{U}=3.387$ & $0.001 * *$ \\
\hline
\end{tabular}

K-W: Kruskal-Wallis test, U: Mann -Whitney test, **: highly significant, p1: between mild and moderate, p2: between mild and severe, p3: between mild and fulminant, p4: between moderate and severe, p5: between moderate and fulminant, p6: between severe and fulminant. Moreover, $\mathrm{H}$ score values of TNF- $\alpha$ expression by inflammatory cells were positively correlated with CRP, ESR, and fecal calprotectin. H score was negatively correlated with Hb level (Table 5). 
Table (5): Correlation between $H$ score values of TNF- $\alpha$ expression in inflammatory cells in IBD cases and age, CRP, Hb, ESR, and fecal calprotectin

\begin{tabular}{||l|c|c|}
\hline \multirow{2}{*}{\multicolumn{1}{|c|}{ Clinicopathological data }} & \multicolumn{2}{|c|}{ H-score in inflammatory bowel disease cases } \\
\cline { 2 - 3 } & Test of sig. & P value \\
\hline Age (years) & $\mathrm{r}=-0.1$ & 0.5 \\
\hline CRP $(\mathbf{m g} / \mathbf{d L})$ & $\mathrm{r}=0.7$ & $<\mathbf{0 . 0 0 1 * *}$ \\
\hline Hb $(\mathbf{g m} / \mathbf{d L})$ & $\mathrm{r}=0.6$ & $<\mathbf{0 . 0 0 1} * *$ \\
\hline ESR $(\mathbf{m m} / \mathbf{h})$ & $\mathrm{r}=0.7$ & $<\mathbf{0 . 0 0 1} * *$ \\
\hline Fecal calprotectin & $\mathrm{r}=0.7$ & $<\mathbf{0 . 0 0 1} * *$ \\
\hline
\end{tabular}

r: Pearson correlation coefficient, **highly significant

There was significant progressive increase of $\mathrm{H}$ score values of TNF- $\alpha$ expression by inflammatory cells with the clinical severity and given treatment (Table 6).

Table (6): Comparison between $H$ score values of TNF- $\alpha$ expression in inflammatory cells in IBD cases as regard disease severity and treatment

\begin{tabular}{|c|c|c|c|c|}
\hline \multirow[t]{2}{*}{ Clinicopathological data } & \multicolumn{2}{|c|}{$\begin{array}{c}\text { H-score of TNF-a expression in } \\
\text { inflammatory cells in bowel disease } \\
\text { cases }\end{array}$} & \multirow[t]{2}{*}{ Test of sig. } & \multirow[t]{2}{*}{$P$ value } \\
\hline & $\mathbf{N}$ & Mean \pm SD & & \\
\hline $\begin{array}{l}\text { Clinical severity: } \\
\text { Mild } \\
\text { Moderate } \\
\text { Severe } \\
\text { Fulminant }\end{array}$ & $\begin{array}{l}14 \\
16 \\
9 \\
2\end{array}$ & $\begin{array}{c}54.3 \pm 8.7 \\
149.4 \pm 9.4 \\
158.9 \pm 4.4 \\
270\end{array}$ & $\mathrm{~K}-\mathrm{W}=23.819$ & $\begin{array}{l}<\mathbf{0 . 0 0 1} * * \\
\mathrm{P} 1:<0.001 \\
\mathrm{P} 2: 0.002 \\
\mathrm{P} 3:<0.001 \\
\mathrm{P} 4: 0.999 \\
\mathrm{P} 5:<0.001 \\
\mathrm{P} 6: 0.002\end{array}$ \\
\hline $\begin{array}{l}\text { Treatment: } \\
\text { Traditional } \\
\text { Anti-TNF therapy after traditional }\end{array}$ & $\begin{array}{l}26 \\
15 \\
\end{array}$ & $\begin{array}{c}84.6 \pm 1.1 \\
194.7 \pm 6.9 \\
\end{array}$ & $\mathrm{U}=4.277$ & $<0.001 * *$ \\
\hline
\end{tabular}

K-W: Kruskal-Wallis test, U: Mann-Whitney test, **: highly significant, p1: between mild and moderate, p2: between mild and severe, $\mathrm{p} 3$ : between mild and fulminant, p4: between moderate and severe, $\mathrm{p} 5$ : between moderate and fulminant, $\mathrm{p} 6$ : between severe and fulminant.

The results of the diagnostic validity of TNF- $\alpha$ expression in IBD, UC, and CD are shown in table 7.

Table (7): Diagnostic validity of TNF- $\alpha$ - expression in IBD cases, UC and CD

\begin{tabular}{||c|c|c|c|}
\hline $\begin{array}{c}\text { TNF diagnostic } \\
\text { validity (control } \\
\mathbf{n = 1 5}\end{array}$ & $\begin{array}{c}\text { IBD Cases } \\
(\mathbf{n = 4 1 )}\end{array}$ & $\begin{array}{c}\text { UC } \\
(\mathbf{n = 2 6 )})\end{array}$ & $\begin{array}{c}\text { CD } \\
(\mathbf{n = 1 5})\end{array}$ \\
\hline Sensitivity & $100 \%$ & $100 \%$ & $100 \%$ \\
\hline Specificity & $73 \%$ & $73 \%$ & $73 \%$ \\
\hline PPV & $91 \%$ & $87 \%$ & $79 \%$ \\
\hline NPV & $100 \%$ & $100 \%$ & $100 \%$ \\
\hline Accuracy & $93 \%$ & $90 \%$ & $87 \%$ \\
\hline
\end{tabular}

There was a significant correlation between response to anti-TNF treatment and TNF- $\alpha$ staining intensity, percentage of positive inflammatory cells expressing TNF- $\alpha$, and H score (Table 8). 
Table (8): Correlation between response to anti-TNF treatment and tumor necrosis factor alpha immunostaining results

\begin{tabular}{|c|c|c|c|c|c|c|}
\hline \multirow{3}{*}{ TNF- $\alpha$ data } & \multicolumn{4}{|c|}{ Anti-TNF treatment $(\mathrm{N}=15)$} & \multirow{3}{*}{ Test of sig. } & \multirow{3}{*}{ P-value } \\
\hline & \multicolumn{2}{|c|}{$\begin{array}{c}\text { Good response } \\
(\mathrm{N}=13)\end{array}$} & \multicolumn{2}{|c|}{$\begin{array}{c}\text { Poor response } \\
(\mathrm{N}=2)\end{array}$} & & \\
\hline & $\mathbf{N}$ & $\%$ & $\mathbf{N}$ & $\%$ & & \\
\hline $\begin{array}{l}\text { TNF- } \boldsymbol{\alpha} \text { expression: } \\
\text { Positive } \\
\text { Negative }\end{array}$ & $\begin{array}{c}13 \\
0\end{array}$ & $\begin{array}{c}100 \\
0\end{array}$ & $\begin{array}{l}2 \\
0\end{array}$ & $\begin{array}{c}100 \\
0\end{array}$ & $X^{2}=0$ & 1 \\
\hline $\begin{array}{l}\text { Staining Intensity } \\
\text { Mild } \\
\text { Moderate } \\
\text { Strong }\end{array}$ & $\begin{array}{c}0 \\
1 \\
12\end{array}$ & $\begin{array}{c}0 \\
7.7 \\
92.3\end{array}$ & $\begin{array}{l}1 \\
1 \\
0\end{array}$ & $\begin{array}{c}50 \\
50 \\
0\end{array}$ & $X^{2}=10.673$ & $0.005 *$ \\
\hline $\begin{array}{l}\text { Percent of positive } \\
\text { inflammatory cells }(\%) \text { : } \\
\text { Mean } \pm \text { SD }\end{array}$ & \multicolumn{2}{|c|}{$74.6 \pm 10.5$} & \multicolumn{2}{|c|}{$55 \pm 7.1$} & $\mathrm{t}=2.513$ & $0.026 *$ \\
\hline $\begin{array}{l}\text { H-score: } \\
\text { Mean } \pm \text { SD }\end{array}$ & \multicolumn{2}{|c|}{$212.3 \pm 41.7$} & \multicolumn{2}{|c|}{$80 \pm 28.3$} & $\mathrm{U}=2.24$ & $0.025 *$ \\
\hline
\end{tabular}

T: T-test, U: Mann-Whitney test * significant

\section{DISCUSSION}

Several biomarkers have been studied with a focus on the degree of correlation with endoscopic disease in IBD. The most commonly used biomarkers include ESR, CRP, FC, and FL ${ }^{(9)}$. Identification of patients that will respond to anti-TNF $\alpha$ agents remains a major clinical challenge. In addition, there is a need to better understand the role of TNF $\alpha$ in these conditions, especially at the inflamed intestinal segments level, to establish why different therapeutic responses are observed in different subjects. Unfortunately, studies addressing this issue are scarce ${ }^{(\mathbf{1 0})}$.

In the current study, CRP (C-reactive protein) levels were significantly higher in patients with IBD and in both UC and CD compared to control. This is agreed with Chen $\boldsymbol{e t}$ al. (11) who conducted a retrospective study that involved 876 IBD patients (275 patients with UC and 601 patients with $\mathrm{CD}$ and reported that CRP levels in active IBD patients were significantly higher.

Moreover, the present study showed that ESR was significantly higher in patients with active IBD. This agreed with Mak et $\boldsymbol{a l} .{ }^{(\mathbf{1 2})}$ who reported that ESR levels were significantly higher and $\mathrm{Hb}$ levels were significantly lower.

There was a significant difference between IBD cases and control regarding TNF- $\alpha$ expression, percentage of positive inflammatory cells, and $\mathrm{H}$ score of TNF. This coincides with Villanacci et al. ${ }^{(\mathbf{1 0})}$ who found that TNF- $\alpha$ was expressed in all inflamed IBD patients and there was strong association between the grade of inflammation and TNF- $\alpha$ positivity.

In the current study, we found that TNF- $\alpha$ was expressed in all patients with IBD and that the percentage of positive inflammatory cells expressing TNF- $\alpha$ and $\mathrm{H}$ score of TNF- $\alpha$ correlated with the clinical severity, histopathology score in UC, and also with endoscopic severity. Villanacci et al. ${ }^{(10)}$ and Olsen et al. (13) reported that TNF- $\alpha$ was highly expressed in UC and correlated to the grade of inflammation.

Moreover, the present study showed that increased percentage of positive inflammatory cells and $\mathrm{H}$ score of TNF- $\alpha$ were significantly associated with the need to give additional treatment other than traditional or conventional therapy with good response to anti-TNF therapy in most patients with increased TNF- $\alpha$ expression. This agreed with Atreya et al. (6) who reported that patients with high numbers of membranebound TNF (mTNF) +ve cells showed significantly higher response rates (92\%) upon subsequent anti-TNF therapy as compared to patients with low amounts of membrane-bound TNF (mTNF) +ve cells. Also, Jessen et al. ${ }^{(14)}$ reported that production of a high level of TNF$\alpha$ by PBMCs from patients with IBD can identify those most likely to have a clinical response to infliximab therapy. Baseline PBMCs from responders produced significantly more TNF- $\alpha$ than from nonresponders.

In the present study, there was a statistically significant positive expression of TNF- $\alpha$ in IBD cases with $100 \%$ sensitivity, $73 \%$ specificity, $91 \%$ PPV, $100 \%$ NPV, and 93\% accuracy. This agreed with Atreya et al. ${ }^{(6)}$ who reported that the sensitivity, specificity and accuracy of TNF- $\alpha$ for the prediction of therapeutic responses were $92 \%, 85 \%$ and $88 \%$, respectively.

\section{CONCLUSION AND RECOMMENDATIONS}

Tumor necrosis factor (TNF) $\alpha$ increased expression is associated with the clinical, endoscopic and histologic severity and is correlated with other markers as CRP, ESR, and FC. Moreover, TNF- $\alpha$ increased expression is associated with the need to give further treatment other than the traditional or 
conventional treatment as anti-TNF therapy with better response along with increased expression, but further studies are needed to compare the tissue and blood expression of TNF- $\alpha$.

Further multicenter studies on bigger number of non-responsive patients are needed with the need to study other pathways of inflammation especially in patients with low expression of TNF- $\alpha$ and identify other markers for response to therapy.

\section{REFERENCES}

1. Khor B, Gardet A, Xavier R (2011): Genetics and pathogenesis of inflammatory bowel disease. Nature, 474:307-317.

2. Neurath M (2014): Cytokines in inflammatory bowel disease. Nat Rev Immunol., 14:329-342.

3. Lopetuso L, Gerardi V, Papa V et al. (2017): Can we predict the efficacy of anti-TNF- $\alpha$ agents? Int J Mol Sci., 18(9):1973.

4. Vermeire S, Van Assche G, Rutgeerts P (2004): Creactive protein as a marker for inflammatory bowel disease. Inflamm Bowel Dis., 10:661-665.

5. Olsen $T$, Rismo $R$, Gundersen $M$ et al. (2016): Normalization of mucosal tumor necrosis factor- $\alpha$ : a new criterion for discontinuing infliximab therapy in ulcerative colitis. Cytokine, 79:90-95.

6. Atreya R, Neumann H, Neufert C et al. (2014): In vivo imaging using fluorescent antibodies to tumor necrosis factor predicts therapeutic response in Crohn's disease. Nat Med., 20:313-318.

7. Geboes K, Riddell R, Ost A et al. (2000): A reproducible grading scale for histological assessment of inflammation inulcerative colitis. Gut, 47(3): 404-9.
8. Chang H, Jung W, Kim A et al. (2017): Expression and prognostic significance of programmed death protein 1 and programmed death liganed-1, and cytotoxic $\mathrm{T}$ lymphocytes-associated molecule- 4 in hepatoceelular carcinoma. APMIS., 125(8):690-698.

9. Menees S, Powell C, Kurlander J et al. (2015): A meta-analysis of the utility of C-reactive protein, erythrocyte sedimentation rate, fecal calprotectin, and fecal lactoferrin to exclude inflammatory bowel disease in adults with IBS. Am J Gastroenterol., 110(3):444454.

10. Villanacci V, Cadei M, Lanzarotto F et al. (2019): Localization of TNF alpha in ileocolonic biopsies of patients with inflammatory bowel disease. Annals of Diagnostic Pathology, 38:20-25.

11. Chen Y, Wang L, Feng SY et al. (2020): The Relationship between C-reactive protein/albumin ratio and disease activity in patients with inflammatory bowel disease. Gastroenterology Research and Practice, 2020: 1-8.

12. Mak L, Tong T, Cheung $K$ et al. (2020): Combined use of common fecal and blood markers for detection of endoscopically active inflammatory bowel disease. Clin Transl Gastroenterol., 11(3): 138.

13. Olsen T, Goll R, Cui G et al. (2007): Tissue levels of tumor necrosis factor-alpha correlates with grade of inflammation in untreated ulcerative colitis. Scand $\mathbf{J}$ Gastroenterol., 42(11):1312-20.

14. Jessen B, Rodriguez Sillke Y, Sonnenberg E et al. (2020): Level of tumor necrosis factor production by stimulated blood mononuclear cells can be used to predict response of patients with inflammatory bowel diseases to infliximab. Clinical Gastroenterology and Hepatology, 19(4):721-731. 